\title{
Analysis of teaching satisfaction based on Markov chain \\ QI ZHAO
}

Department of Foundation, Shandong Yingcai University, Jinan 250104, China

zaho231200@163.com

Keywords: Markov chain, teaching satisfaction, probability transition matrix

Abstract. This paper introduces Markov process of time discrete and countable state,i.e. Markov chain. Elaborated how Analysis of teaching satisfaction using Markov chain methods, construct Markov chain using teaching satisfaction over the years, construct probability transition matrix. After proper treatment, To extract the useful information, To provide a theoretical basis for the improvement of teaching quality

\section{Introduction}

The establishment of evaluation system of teaching quality, to improve the quality of education and teaching has positive significance.How to establish a scientific and effective teaching quality evaluation method, is an important problem faced by the schools.In this paper, by using the theory of Markov chain, put forward an evaluation method of teaching satisfaction

\section{Basic knowledge of Markov chain}

Study on change rule of time series via Markov prediction method is an important application of the theory of Markov chain. So, first we shall introduce Basic knowledge of Markov chain

Let $\left\{X_{t}: t \geq 0\right\}$ denote Sequence of random variables, let $S$ denote state space, i. e the range of possible values. The random variable is a Markov chain if the transition probabilities between different values in the state space depend only on the random variable's current state, i.e. If for $\forall t \geq 0$ and any state $s_{i}, s_{j}, s_{i_{0}}, \ldots . . s_{i_{t-1}}$ The following are established

$$
P\left(X_{t+1}=s_{j} \mid X_{t}=s_{i}, X_{t-1}=s_{i_{t-1}}, \ldots . . X_{0}=s_{i_{0}}\right)=P\left(X_{t+1}=s_{j} \mid X_{t}=s_{i}\right)
$$

Markov chain is a Markov process of time discrete and countable state, intuitively, for a Markov random variable the only information about the past needed to predict the future is the current state of the random variable, And not related to previous state.Properties of Markov chain is completely decided by its transition probability (or the transition kernel). let $P(i, j)$ denote the probability that a process at state space $s_{i}$ moves to state $s_{j}$ in a single step, i.e.

$$
P(i, j)=P(i \rightarrow j)=P\left(X_{t+1}=s_{j} \mid X_{t}=s_{i}\right)
$$

Definition 1.1 If $P\left(X_{t+1}=s_{j} \mid X_{t}=s_{i}\right)=P\left(X_{1}=s_{j} \mid X_{0}=s_{i}\right)$, then we call the Markov chain is time homogeneous.

This paper discussed is this

Let

$$
\pi_{j}(t)=P\left(X_{t}=s_{j}\right)
$$

Denote the probability that the chain is in state $s_{j}$ at time $t$ and let $\pi(t)$ denote the row vector of the state space probabilities at step $t$.let $\pi(0)$ denote starting vector. often all the element of $\pi(0)$ are zero except for a single element of 1,corresponding to the process starting in that particular state. As the chain progress, the probability values get spread out over the possible state space.

The probability that the chain has state value $s_{i}$ at time $t+1$ is given by the Chapman-Kolomogrov equation 


$$
\pi_{i}(t+1)=P\left(X_{t+1}=s_{i}\right)=\sum_{k} P\left(X_{t+1}=s_{i} \mid X_{t}=s_{k}\right) P\left(X_{t}=s_{k}\right)=\sum_{k} P(k, i) \pi_{k}(t)
$$

Successive iteration of the Chapman-Kolomogrov equation describes the evolution of the Markov chain.

Definition 1.2 If a matrix whose $i, j$ th element is $P(i, j)$, Then we call the matrix is probability transition matrix

$P(i, j)$ is the probability of moving from state $s_{i}$ to $s_{j}$, this implies that each rows sum to 1 ,

$$
\text { as } \sum_{j} P(i, j)=\sum_{j} P(i \rightarrow j)=1
$$

Then the Chapman-Kolomogrov equation can be written in matrix form is more compact

$$
\pi(t+1)=\pi(t) P
$$

Using the matrix form, we can iterate the Chapman-Kolomogrov equation, as

$$
\pi(t)=\pi(t-1) P=(\pi(t-2) P) P=\pi(t-2) P^{2}
$$

Continuing in this fashion shows that

$$
\pi(t)=\pi(0) P^{t}
$$

Defining the n-step transition probability $P_{i j}{ }^{(n)}$ as the probability that the process is in state $s_{j}$ given that it started in state $s_{i}$ n steps ago, i.e.,

$$
P_{i j}^{(n)}=P\left(X_{t+n}=s_{j} \mid X_{t}=s_{i}\right)
$$

$P_{i j}^{(n)}$ is the $i, j$ th element of $P^{n}$

Definition 1.3 A Markov chain is irreducible if there exists a positive integer $n_{i j}$ such that $p_{i j}^{n_{i j}}>0$ for all $i, j$

If A Markov chain is irreducible implies all states communicate with each other, as one can always go from any state to any other state (although it may take more than one step)

Definition 1.4 Let $\mathrm{E}$ denote state space of time homogeneous Markov chain $\left\{X_{n}, n \geq 1\right\}$, if there exist constant $\pi_{j}$ independent of $i$ for all state $i, j \in E$,

$$
\lim _{n \rightarrow \infty} p_{i j}^{(n)}=\pi_{j} \quad i, j \in E
$$

Then the time homogeneous Markov chain is ergodic, and $\pi_{j}$ is The steady state probability of state $j$

$$
\begin{array}{r}
\text { Definition 1.5 } \pi^{*} \text { satisfies } \\
\pi^{*}=\pi^{*} P
\end{array}
$$

Then we call $\pi^{*}$ is a stationary distribution

Theorem1.6 Let $E=\{1,2, \ldots, N\}$ denote state space of time homogeneous Markov chain $\left\{X_{n}, n \geq 1\right\}$, if there exists a positive integer $n_{i j}$ such that $p_{i j}^{n_{i j}}>0$ for all $i$, $j$ (i.e. irreducible), the Markov chain is ergodic, and its steady state probability is stationary distribution

Example 1.7 Suppose the state space are (Rain, Sunny, Cloudy) and weather follows a Markov process. Thus, the probability of tomorrow's weather simply depends on today's weather, and not any other previous days. Suppose the probability transitions given today is rainy are

$P$ (Rain tomorrow $\mid$ Rain today $)=0.5$

$P$ (Sunny tomorrow $\mid$ Rain today $)=0.25$ 
$P($ Cloudy tomorrow $\mid$ Rain today $)=0.25$

The first row of the transition probability matrix thus becomes

$(0.5,0.25,0.25)$

Suppose the rest of the transition matrix is given by

$$
\begin{array}{r}
P=\left[\begin{array}{ccc}
0.5 & 0.25 & 0.25 \\
0.5 & 0 & 0.5 \\
0.25 & 0.25 & 0.5
\end{array}\right] \\
P^{(2)}=P^{2}=\left[\begin{array}{ccc}
\frac{7}{16} & \frac{3}{16} & \frac{3}{8} \\
\frac{3}{8} & \frac{1}{4} & \frac{3}{8} \\
\frac{3}{8} & \frac{3}{16} & \frac{7}{16}
\end{array}\right]
\end{array}
$$

The Markov chain is ergodic, and have stationary distribution $\pi^{*}=\left(\pi_{1}, \pi_{2}, \pi_{3}\right)$,

To satisfy the equations. 1

$$
\left\{\begin{array}{l}
\pi_{1}=\frac{1}{2} \pi_{1}+\frac{1}{2} \pi_{2}+\frac{1}{4} \pi_{3} \\
\pi_{2}=\quad \frac{1}{4} \pi_{1}+\frac{1}{4} \pi_{3} \\
\pi_{3}=\frac{1}{4} \pi_{1}+\frac{1}{2} \pi_{2}+\frac{1}{2} \pi_{3}
\end{array}\right.
$$

And $\quad \pi_{i}>0, i=1,2,3, \sum_{i=1}^{3} \pi_{i}=1$

The solution of this equation can be obtained

$$
\left\{\begin{array}{l}
\pi_{1}=\frac{2}{5} \\
\pi_{2}=\frac{1}{5} \\
\pi_{3}=\frac{2}{5}
\end{array}\right.
$$

The basic idea of discrete-state Markov chain can be generalized to a continuous state Markov process, Let $P(x, y)$ denote probability kernel of the Markov process and satisfies

$$
\int P(x, y) d y=1
$$

And the continuous extension of the Chapman-Kologronvo equation becomes

$$
\pi(y)=\int \pi_{t-1}(x) P(x, y) d y
$$

At equilibrium that stationary distribution satisfies

$$
\pi^{*}(y)=\int \pi^{*}(x) P(x, y) d y
$$

In addition, A sufficient condition for a unique stationary distribution is that the detailed balance equation holds,

$$
P(j, k) \pi_{j}^{*}=P(k, j) \pi_{k}^{*} \text { (for all } k \text { and } j \text { ) }
$$

The Markov chain is said to be reversible, and hence the detailed balance equation is also called the reversibility condition.

Let $(\pi P)_{j}$ denote the $j$ th element of $\pi P$ 


$$
(\pi P)_{j}=\sum_{i} \pi_{i} P(i, j)=\sum_{i} \pi_{j} P(j, i)=\pi_{j} \sum_{i} P(j, i)=\pi_{j}
$$

So the reversibility condition implies $\pi=\pi P$

\section{Analysis of teaching satisfaction base on Markov chain}

Construct probability transition matrix In the Markov forecasting method, construct transition probability matrix is very important. There are usually two kinds of method to construct transfer matrix. The first method is subjective probability method, This method is generally lack of historical statistical data, according to the long-term accumulation of experience and understanding of the predicted event, a subjective estimation of the probability of events. The second method is statistical estimation method, This is a kind of frequency is used instead of probability estimation method,_This method requires a large amount of historical data. Because the school each semester will investigate the satisfaction of every teacher, there are plenty of historical data, so we use the statistical estimation method

teachers' satisfaction survey results are presented in the form of percentage, We assume that the satisfaction more than $90 \%$ is very satisfied, denoted as status $1,60 \%-90 \%$ is satisfied, denoted as status 2 , less than $60 \%$ is dissatisfied, denoted as status 3. $P_{i j}$ denote the probability that a process at state space $i$ moves to state $j$ in a single step. Let $n_{i j}$ denote the Statistical number that a process at state space $i$ moves to state $j$ in a single step, the following formula is the estimation formula of $P_{i j}$

$$
P_{i j} \approx \frac{n_{i j}}{\sum_{j=1}^{m} n_{i j}}, i, j=1,2, \ldots, m
$$

Example In the teacher's satisfaction survey, The 24 state transition conditions as follows

$$
\begin{aligned}
& 1 \rightarrow 1,2 ; 2 \rightarrow 1,8 ; 3 \rightarrow 1,0 \\
& 1 \rightarrow 2,6 ; 2 \rightarrow 2,4 ; 3 \rightarrow 2,2 \\
& 1 \rightarrow 3,2 ; 2 \rightarrow 3,0 ; 3 \rightarrow 3,0
\end{aligned}
$$

Transition probability in a single step can be approximately expressed as:

$$
\begin{array}{lll}
P_{11} \approx \frac{2}{2+6+2}=\frac{1}{5} & P_{12} \approx \frac{6}{2+6+2}=\frac{3}{5} & P_{13} \approx \frac{2}{2+6+2}=\frac{1}{5} \\
P_{21} \approx \frac{8}{8+4}=\frac{2}{3} & P_{22} \approx \frac{4}{8+4}=\frac{1}{3} & P_{23} \approx \frac{0}{8+4}=0 \\
P_{31} \approx \frac{0}{2}=0 & P_{32} \approx \frac{2}{2}=1 & P_{33} \approx \frac{0}{2}=0
\end{array}
$$

Thus, probability transition matrix as follows

$$
P=\left[\begin{array}{ccc}
\frac{1}{5} & \frac{3}{5} & \frac{1}{5} \\
\frac{2}{3} & \frac{1}{3} & 0 \\
0 & 1 & 0
\end{array}\right]
$$




$$
P^{(3)}=P^{3}=\left[\begin{array}{ccc}
\frac{163}{375} & \frac{179}{375} & \frac{33}{375} \\
\frac{278}{675} & \frac{349}{675} & \frac{48}{675} \\
\frac{16}{45} & \frac{23}{45} & \frac{6}{45}
\end{array}\right]
$$

the Markov chain is ergodic, and have stationary distribution Now, we predict the satisfaction of the teacher

Let $P_{1}, P_{2}, P_{3}$ respectively denote the probability of very satisfied, the probability of satisfied , the probability of dissatisfied, There are

Thus obtained the equations. 2

$$
\left(P_{1}, P_{2}, P_{3}\right)=\left(P_{1}, P_{2}, P_{3}\right)\left[\begin{array}{ccc}
\frac{1}{5} & \frac{3}{5} & \frac{1}{5} \\
\frac{2}{3} & \frac{1}{3} & 0 \\
0 & 1 & 0
\end{array}\right]
$$

$$
\left\{\begin{array}{l}
P_{1}=\frac{1}{5} P_{1}+\frac{2}{3} P_{2} \\
P_{2}=\frac{3}{5} P_{1}+\frac{1}{3} P_{2}+P_{3} \\
P_{3}=\frac{1}{5} P_{1}
\end{array}\right.
$$

And $\quad P_{i}>0, i=1,2,3, \sum_{i=1}^{3} P_{i}=1$

The solution of this equation can be obtained

$$
\left\{\begin{array}{l}
P_{1}=\frac{5}{12} \\
P_{2}=\frac{1}{2} \\
P_{3}=\frac{1}{12}
\end{array}\right.
$$

\section{Conclusion}

Based on the Markov chain analysis teaching satisfaction is a dynamic method, Make full use of a large amount of information contained in the transition probability matrix, the evaluation results are more objective and true. This method is also applicable to other quantitative appraisal, has the widespread application prospect

\section{References}

[1] G. L. Gong , M. P. Qian. The application of stochastic process . Tsinghua University press, Beijing, 2004

[2] Y. Z. Zhang. Study on higher education evaluation method. Fudan University press, Shanghai, 2002

[3] Y.Q. Li, Ch. Liu, Q. Wang. stochastic process. National Defense Industry Press, Beijing, 2009

[4] Walsh, B. (2004). Markov Chain Monte Carlo and Gibbs Sampling. Lecture Notes for 
EEB 581, version 26 April 2004.

[5] Gareth O. Roberts , Jeffrey S . Rosenthal., General state space Markov chains and MCMC algorithms, Probability surveys Vol.1 (2004) 20-71 